\title{
Loss of Single-Stranded DNA Binding Protein 2 Expression Is Associated with Aggressiveness and Poor Overall Survival in Patients with Invasive Breast Carcinoma
}

\author{
Hosub Park ${ }^{1}$, Seungyun Jee ${ }^{1} \mathbb{1}$, Hwangkyu Son ${ }^{1}$, Hyebin Cha ${ }^{1}$, Seongsik Bang ${ }^{1}{ }^{\mathbb{D}}$, Hyunsung Kim ${ }^{1}{ }^{1}$,

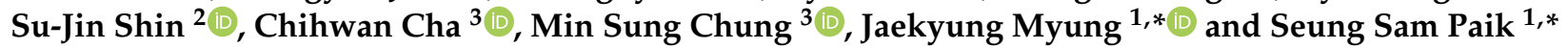

1 Department of Pathology, Seoul Hospital, Hanyang University College of Medicine, Seoul 04763, Korea; parkhstm@gmail.com (H.P.); jee.seung.yun@gmail.com (S.J.); ganzi4900@gmail.com (H.S.); cnbin0111@gmail.com (H.C.); grypony@naver.com (S.B.); hhnt5841@gmail.com (H.K.)

2 Department of Pathology, Gangnam Severance Hospital, Yonsei University College of Medicine, Seoul 06273, Korea; charm@yuhs.ac

3 Department of Surgery, Seoul Hospital, Hanyang University College of Medicine, Seoul 04763, Korea; chachihwan@gmail.com (C.C.); bovie@hanyang.ac.kr (M.S.C.)

* Correspondence: tontos016@naver.com (J.M.); sspaik@hanyang.ac.kr (S.S.P.); Tel.: +82-2-2220-8960 (S.S.P.)

check for updates

Citation: Park, H.; Jee, S.; Son, H.; Cha, H.; Bang, S.; Kim, H.; Shin, S.-J.; Cha, C.; Chung, M.S.; Myung, J.; et al. Loss of Single-Stranded DNA Binding Protein 2 Expression Is Associated with Aggressiveness and Poor Overall Survival in Patients with Invasive Breast Carcinoma.

Diagnostics 2022, 12, 487.

https: / /doi.org/10.3390/

diagnostics12020487

Academic Editors: Gustavo Baldassarre and Joseph Geradts

Received: 30 December 2021 Accepted: 12 February 2022 Published: 14 February 2022

Publisher's Note: MDPI stays neutral with regard to jurisdictional claims in published maps and institutional affiliations.

Copyright: (C) 2022 by the authors. Licensee MDPI, Basel, Switzerland. This article is an open access article distributed under the terms and conditions of the Creative Commons Attribution (CC BY) license (https:// creativecommons.org/licenses/by/ $4.0 /)$.

\begin{abstract}
Background: Single-stranded DNA binding protein 2 (SSBP2) is involved in the DNA damage response and the maintenance of genome stability. Previous studies have suggested that $S S B P 2$ has a tumor suppressor function or oncogenic function. Loss of SSBP2 expression has been reported in various tumors. However, the role of $S S B P 2$ expression in invasive breast carcinoma has not been reported. Methods: Immunohistochemical staining for SSBP2 was performed on tissue microarrays consisting of 491 invasive breast carcinoma cases. The result of nuclear SSBP2 staining was stratified as either negative or positive. Then, we investigated the correlations between SSBP2 expression and various clinicopathological parameters and patient outcomes. Results: Loss of nuclear SSBP2 expression was observed in 61 cases (12.4\%) of 491 invasive breast carcinomas. Loss of nuclear SSBP2 expression was significantly correlated with larger tumor size $(p<0.001$, chi-squared test), higher histological grade ( $p=0.016$, Cochran-Armitage trend test), higher pathological T stage $(p<0.001$, Cochran-Armitage trend test), estrogen receptor status ( $p<0.001$, chi-squared test), and molecular subtype ( $p<0.001$, chi-squared test). Kaplan-Meier survival analysis revealed that patients with loss of nuclear SSBP2 expression had worse overall survival ( $p=0.013$, log-rank test). However, loss of nuclear SSBP2 expression was not correlated with recurrence-free survival $(p=0.175, \log$-rank test). Conclusions: Loss of nuclear SSBP2 expression was associated with adverse clinicopathological characteristics and poor patient outcomes. SSBP2 acts as a tumor suppressor in invasive breast carcinoma and may be used as a prognostic biomarker.
\end{abstract}

Keywords: single-stranded DNA binding protein 2; invasive breast carcinoma; prognosis

\section{Introduction}

Breast cancer is the most common malignant neoplasm and the leading cause of cancer mortality among women in Western countries as well as the leading contributor to the global cancer incidence rate in 2020 [1]. In 2020, estimated new breast cancer cases totaled 2.3 million, or $11.7 \%$ of all new cancer cases, and breast cancer was the fifth leading cause of cancer mortality worldwide, causing 685,000 deaths [1]. Breast cancer is a genetically and clinically heterogeneous disease. Although there have been marked advances in understanding breast cancer development and cancer biology, the specific treatment problem persists [2]. Various clinicopathological parameters, such as histological grade, estrogen receptor (ER), progesterone receptor (PR), human epidermal growth factor receptor 2 (HER2) gene amplification, and American Joint Committee on Cancer (AJCC) 
stage are currently considered in the prognosis and management of breast cancer [3]. Using proven clinicopathological prognostic factors, various proteins have been proposed as potential prognostic biomarkers of breast cancer [4].

Molecular subtype has an important influence on patient management. Molecular subtypes can be classified using surrogate markers of ER, PR, HER2, and Ki-67 immunostaining. The luminal subtype with positive ER and PR has a better prognosis than the HER2 or triple negative subtypes. Luminal subtype is divided into luminal A subtype, which has a good prognosis, and luminal $\mathrm{B}$ subtype, which has relatively a poor prognosis. Luminal B subtype shows a relatively high Ki-67 index compared to luminal A subtype. Some groups of luminal B subtype show HER2 positivity. The HER2 subtype is ER-negative and has HER2 overexpression. This subtype can be treated by target therapy for HER2. Triple negative subtype is negative for ER, PR, and HER2, and this subtype has a worst prognosis [5].

The single-stranded DNA binding protein 2 (SSBP2) gene, which is located at chromosome 5q14.1, was previously identified as a candidate tumor suppressor in myeloid leukemia patients [6]. The SSBP2 gene is a subunit of the ssDNA-binding complex and is involved in the maintenance of hematopoietic stem cells and the maintenance of genome stability [6,7]. SSBP2 binds to the transcriptional adaptor protein, Lim domain binding protein 1 (LDB1), through a highly conserved $\mathrm{N}$-terminal domain and enhances LDB1 stability to regulate gene expression [8]. LDB1 is a protein that is reported to be involved in tumorigenesis in leukemia, head and neck cancer, and colon cancer [9]. The role of SSBP2 in human malignancies has been studied in several solid tumors and myeloid leukemia [10-16]. Regarding whether SSBP2 function is a tumor suppressor or tumor promoter, its exact role remains unclear. Several studies have demonstrated that SSBP2 is a tumor suppressor in solid tumors and myeloid leukemia [10,11,14-16]. However, a few studies have suggested that SSBP2 is a tumor promoter in glioblastoma and hepatocellular carcinoma [12,13]. The role of SSBP2 in human breast cancer has not yet been reported.

In this study, we investigated the expression of SSBP2 by immunohistochemistry in invasive breast carcinoma tissues, analyzed the associations between SSBP2 expression and various clinicopathological characteristics, and assessed whether SSBP2 is a prognostic factor for patient survival.

\section{Materials and Methods}

\subsection{Patients and Tumor Samples}

We enrolled a consecutive series of 541 patients with invasive breast carcinoma. All cases were diagnosed and underwent surgery at Hanyang University Hospital (Seoul, South Korea) between February 2003 and January 2017. We excluded patients with incomplete clinical follow-up data or no available paraffin blocks and started the study with 491 cases of invasive breast carcinoma, consisting of 471 invasive breast carcinomas of no special type, 19 invasive lobular carcinomas, and 1 case of adenoid cystic carcinoma. The baseline characteristics of included patients are summarized in Table 1 . The mean age of patients was 52.9 years, and the mean follow-up period was 77 months. Of the included 491 cases, 104 cases were histological grade 1, 225 cases were histological grade 2, and 162 cases were histological grade 3 . According to the eighth edition of the AJCC system, 183 cases were stage I, 207 cases were stage II, 92 cases were stage III, and 9 cases were stage IV. All tissue samples were fixed in formalin and embedded in paraffin. We reviewed all slides stained with hematoxylin and eosin (H\&E) together with pathology reports and other medical records. The assessed clinicopathological characteristics were patient age, tumor size, histological grade, pathological T (pT) stage, pathological N (pN) stage, AJCC stage, lymph node metastasis, distant metastasis, ER and PR status, HER2 status, molecular subtype, and patient survival. This study was approved by the Institutional Review Board of the Hanyang University Hospital (HYUH 2021-12-014-001), and the requirement to collect informed consent was waived. 
Table 1. Baseline characteristics of enrolled patients $(n=491)$.

\begin{tabular}{|c|c|}
\hline Clinicopathological Characteristics & Value (\%) \\
\hline Age (years, median, mean $\pm S D$ ) & $51,52.9 \pm 11.1$ \\
\hline \multicolumn{2}{|l|}{ Size } \\
\hline$<2 \mathrm{~cm}$ & $226(46.0 \%)$ \\
\hline$>2 \mathrm{~cm}$ & $265(54.0 \%)$ \\
\hline \multicolumn{2}{|l|}{ Histological grade } \\
\hline 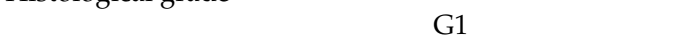 & $104(21.2 \%)$ \\
\hline G2 & $225(45.8 \%)$ \\
\hline G3 & $162(33.0 \%)$ \\
\hline \multicolumn{2}{|l|}{ pT stage } \\
\hline $\mathrm{T} 1$ & $226(46.0 \%)$ \\
\hline $\mathrm{T} 2$ & $223(45.4 \%)$ \\
\hline $\mathrm{T} 3$ & $28(5.7 \%)$ \\
\hline $\mathrm{T} 4$ & $14(2.9 \%)$ \\
\hline \multicolumn{2}{|l|}{ pN stage } \\
\hline $\begin{array}{l}\text { N0 } \\
\text { N1 }\end{array}$ & $\begin{array}{l}307(62.5 \%) \\
108(22.0 \%)\end{array}$ \\
\hline N2 & $\begin{array}{l}108(22.0 \%) \\
41(8.4 \%)\end{array}$ \\
\hline N3 & $35(7.1 \%)$ \\
\hline \multicolumn{2}{|l|}{ AJCC stage } \\
\hline I & $183(37.3 \%)$ \\
\hline II & $207(42.2 \%)$ \\
\hline III & $92(18.7 \%)$ \\
\hline IV & $9(1.8 \%)$ \\
\hline \multicolumn{2}{|l|}{ LN metastasis } \\
\hline Negative & $307(62.5 \%)$ \\
\hline Positive & $184(37.5 \%)$ \\
\hline \multicolumn{2}{|l|}{ Distant metastasis } \\
\hline Negative & $482(98.2 \%)$ \\
\hline Positive & $9(1.8 \%)$ \\
\hline \multicolumn{2}{|l|}{ ER status } \\
\hline Negative & $192(39.1 \%)$ \\
\hline Positive & $299(60.9 \%)$ \\
\hline \multicolumn{2}{|l|}{ PR status } \\
\hline Negative & $199(40.5 \%)$ \\
\hline Positive & $292(59.5 \%)$ \\
\hline \multicolumn{2}{|l|}{ HER2 status } \\
\hline Negative & $338(68.9 \%)$ \\
\hline \multirow{2}{*}{\multicolumn{2}{|c|}{ Molecular subtype }} \\
\hline & \\
\hline Luminal A & $200(40.7 \%)$ \\
\hline Luminal B & $108(22.0 \%)$ \\
\hline HER2 & $76(15.5 \%)$ \\
\hline TNBC & $107(21.8 \%)$ \\
\hline
\end{tabular}

Abbreviations: SD, standard deviation; AJCC, American Joint Committee on Cancer; LN, lymph node; ER, estrogen receptor; PR, progesterone receptor; HER2, human epidermal growth factor receptor 2; TNBC, triple-negative breast carcinoma.

\subsection{Tissue Microarray (TMA) Construction}

A manual tissue microarrayer (Unitma, Seoul, South Korea) was used for TMA construction from archival formalin-fixed and paraffin-embedded tissue blocks. The most representative non-necrotic central portion of the tumor was selected by light microscopy. We punched a tissue cylinder $3 \mathrm{~mm}$ in diameter from a previously marked lesion of each donor block and transferred it to the recipient block (Unitma, Seoul, South Korea). Each TMA block comprised $6 \times 5$ samples.

\subsection{Immunohistochemical (IHC) Staining}

We performed the IHC staining for SSBP2 on $4 \mu \mathrm{m}$ thick sections from the TMA blocks. All TMA sections were deparaffinized in xylene. The deparaffinized sections were then rehydrated by a series of 5 min washes in 100\%, 90\%, and 75\% ethanol and phosphate-buffered saline (PBS). To retrieve the antigen, the sections were heated in sodium citrate buffer $(\mathrm{pH}, 6.0)$ in an autoclave at $100{ }^{\circ} \mathrm{C}$ for $20 \mathrm{~min}$. Then, we blocked endogenous peroxidase activity with peroxidase blocking solution (S2023; Dako, Glostrup, Denmark). The TMA slides were incubated with a rabbit monoclonal SSBP2 antibody (1:100 dilution, 
ab177944; Abcam, Cambridge, UK) at $4{ }^{\circ} \mathrm{C}$ overnight, then incubated with a labeled polymer (EnVision/HRP, K5007; Dako, Glostrup, Denmark) for 30 min at room temperature. Monoclonal mouse anti-ER (Novocastra Laboratories, Newcastle, UK), monoclonal mouse anti-PR (Novocastra Laboratories, Newcastle, UK), monoclonal mouse anti-c-erbB-2 (Novocastra Laboratories, Newcastle, UK), and monoclonal mouse Ki-67 antibody (Novocastra Laboratories, Newcastle, UK) were diluted 1:50, 1:100, 1:800, and 1:100 in goat serum, respectively. Next, 3,3'-diaminobenzidine tetrahydrochloride was used as a chromogen for visualization, and counterstained with Mayer's hematoxylin.

\subsection{Interpretation of IHC Staining and Molecular Subtypes}

SSBP2 expression was evaluated according to the nuclear staining extent of tumor cells using a light microscope by two pathologists (H.P. and S.P.) who were blinded to the clinicopathological parameters and the patient clinical outcomes. According to previous reports $[14,16]$, we subdivided the patients into a positive subgroup (proportion of positive tumor cells $>10 \%$ of the total tumor cells) and a negative subgroup (proportion of positive tumor cells $<10 \%$ of the total tumor cells).

ER and PR status was interpreted by Allred score for nuclear staining, according to ASCO/CAP guidelines. Intensity scores of $0,1,2$, and 3 were given. Score 0 was completely negative and score 3 was strong. A proportion score of 0 to 5 was given to $0 \%,<1 \%, 1-10 \%$, $11-33 \%, 34-66 \%$, and $67-100 \%$, respectively. When the sum of the intensity score and proportion score was 0 and 2, it was interpreted as negative, and when the sum of those scores was 3 or more, it was interpreted as positive.

HER2 status was interpreted according to the ASCO/CAP guidelines. The case where strong membranous stain was observed in more than $10 \%$ of cells in immunohistochemical staining was interpreted as positive. When weak to moderate membranous stain was observed, dual probe SISH was performed. In the SISH, HER2/CEP17 ratio of 2 or more and HER2 signal of 4 or more per cell were interpreted as positive.

$\mathrm{Ki}-67$ was read by eyeballing the percentage of cells showing nuclear stain from $0 \%$ to $100 \%$ in $10 \%$ increments.

Molecular subtype was classified by applying ER, PR, and HER2 status as surrogate markers. Cases with positive ER status were classified as "luminal", and cases with negative ER status and positive HER2 status were classified as "HER2-positive". Cases which were negative for HER2, ER, and PR were classified as "triple negative". Among the luminal subtypes, the cases with more than $10 \%$ of Ki-67 rate or the positive HER2 status were classified as "luminal B", and the cases which did not satisfy both conditions were classified as "luminal A". The cut-off point of the Ki-67 labeling index that distinguishes luminal A and $B$ was presented at various values between $10 \%$ and $20 \%$ in previous studies [17]. In this study, the cut-off point was set at $10 \%$ for conservative evaluation.

\subsection{Statistical Analysis}

For statistical analysis, Pearson's chi-squared test, Student's $t$ test, and the CochranArmitage trend test were used to evaluate any potential association between SSBP2 expression and the clinicopathological parameters in categorical variables. Overall survival (OS) was defined as the duration from surgical treatment to death, and recurrence-free survival (RFS) was defined as the duration from surgical treatment to the first recurrence, either clinically or pathologically. The Kaplan-Meier method with a log-rank test was used to construct survival curves, and univariate and multivariate Cox proportional hazard ratio models were used to determine the significant prognostic variables. $p$ values $<0.05$ were regarded as statistically significant. Statistical analysis was performed using $\mathrm{R}$ version 3.6.2 (R Foundation for Statistical Computing, Vienna, Austria). 


\section{Results}

\subsection{Patterns of SSBP2 Expression}

SSBP2 expression was evaluated on TMA slides by two pathologists. Adjacent normal breast ductal epithelial cells showed intact nuclear SSBP2 expression. Of 491 invasive breast carcinoma cases, 61 cases (12.4\%) showed negative nuclear SSBP2 expression and 430 cases $(87.6 \%)$ showed positive nuclear SSBP2 expression on IHC staining. Representative microscopic photographs are shown in Figure 1.
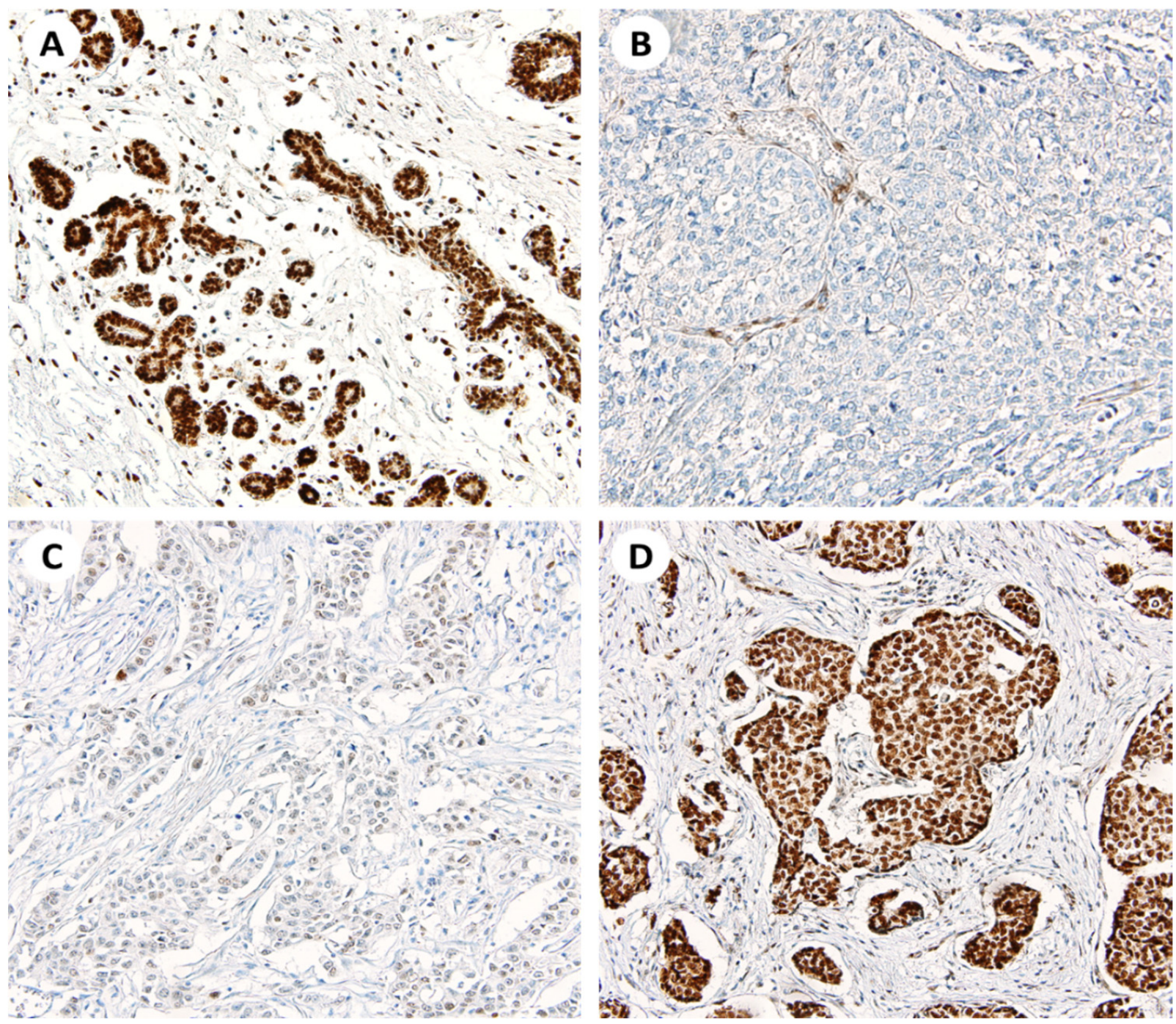

Figure 1. Representative photomicrographs of $S S B P 2$ IHC stain. (A) Positive nuclear stain on adjacent normal breast ductal epithelial cells $(\times 200)$. (B) Negative stain on the tumor cells $(\times 200)$. (C) Weak nuclear positivity on the tumor cells $(\times 200)$. (D) Strong nuclear positivity on the tumor cells $(\times 200)$.

\subsection{Correlations between Nuclear SSBP2 Expression and Clinicopathological Parameters}

The correlations between nuclear SSBP2 expression and clinicopathological parameters are summarized in Table 2. Negative nuclear SSBP2 expression was significantly correlated with larger tumor size $(p<0.001$, chi-squared test), higher histological grade $(p=0.016$, Cochran-Armitage trend test), higher $\mathrm{pT}$ stage $(p<0.001$, Cochran-Armitage trend test), ER status ( $p<0.001$, chi-squared test), and molecular subtype ( $p<0.001$, chi-squared test). There was no statistically significant correlation between nuclear SSBP2 expression and age, $\mathrm{pN}$ stage, AJCC stage, lymph node metastasis, distant metastasis, PR status, or HER2 status. Cases of the pT1 stage were analyzed by sub-stratification into T1a, T1b, and T1c. In the SSBP2-positive group, 213 pT1 cases were distributed in $8(3.8 \%), 30(14.1 \%)$, and 
$175(82.2 \%)$ cases in $\mathrm{T} 1 \mathrm{a}, \mathrm{T} 1 \mathrm{~b}$, and T1c, respectively. In the SSBP2-negative group, 13 cases of pT1 were distributed as $0(0 \%), 2(15.4 \%)$, and $11(84.6 \%)$, respectively. There was no significant trend in T1a, b, or c stages according to SSBP2 expression ( $p$ value $=0.896$, Cochran-Armitage trend test).

Table 2. Correlations between SSBP2 expression and clinicopathological parameters $(n=491)$.

\begin{tabular}{|c|c|c|c|}
\hline \multirow[b]{2}{*}{ Parameter } & \multicolumn{2}{|c|}{ SSBP2 Expression } & \multirow[b]{2}{*}{$p$ Value } \\
\hline & $\begin{array}{c}\text { Positive }(n=430) \\
\text { No. }(\%)\end{array}$ & $\begin{array}{c}\text { Negative }(n=61) \\
\text { No. }(\%)\end{array}$ & \\
\hline Age (years, mean \pm SD) & & & $0.427^{\dagger}$ \\
\hline & $52.7 \pm 10.9$ & $54 \pm 12.1$ & \\
\hline Size & & & $<0.001$ \\
\hline$\leq 2 \mathrm{~cm}$ & $210(48.8 \%)$ & $16(26.2 \%)$ & \\
\hline$>2 \mathrm{~cm}$ & $220(51.2 \%)$ & $45(73.8 \%)$ & \\
\hline Histological grade & & & 0.016 * \\
\hline G1 & $95(22.1 \%)$ & $9(14.8 \%)$ & \\
\hline $\mathrm{G} 2$ & $202(47.0 \%)$ & $23(37.7 \%)$ & \\
\hline G3 & $133(30.9 \%)$ & $29(47.5 \%)$ & \\
\hline pT stage & & & $0.001 *$ \\
\hline $\mathrm{T} 1$ & $213(49.6 \%)$ & $13(21.3 \%)$ & \\
\hline $\mathrm{T} 2$ & $182(42.3 \%)$ & $41(67.2 \%)$ & \\
\hline T3 & $25(5.8 \%)$ & $3(4.9 \%)$ & \\
\hline $\mathrm{T} 4$ & $10(2.3 \%)$ & $4(6.6 \%)$ & \\
\hline pN stage & & & 0.958 * \\
\hline No & $269(62.6 \%)$ & $38(62.3 \%)$ & \\
\hline N1 & $94(21.9 \%)$ & $14(23.3 \%)$ & \\
\hline $\mathrm{N} 2$ & $37(8.6 \%)$ & $4(6.6 \%)$ & \\
\hline N3 & $30(7.0 \%)$ & $5(8.2 \%)$ & \\
\hline AJCC stage & & & 0.053 * \\
\hline 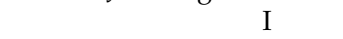 & $170(39.5 \%)$ & $13(21.3 \%)$ & \\
\hline II & $172(40.0 \%)$ & $35(57.4 \%)$ & \\
\hline III & $81(18.8 \%)$ & $11(18.0 \%)$ & \\
\hline IV & $7(1.6 \%)$ & $2(3.3 \%)$ & \\
\hline LN metastasis & & & 0.968 \\
\hline Negative & $269(62.6 \%)$ & $38(62.3 \%)$ & \\
\hline Positive & $161(37.4 \%)$ & $23(37.7 \%)$ & \\
\hline Distant metastasis & & & 0.368 \\
\hline Negative & $423(98.4 \%)$ & $59(96.7 \%)$ & \\
\hline Positive & $7(1.6 \%)$ & $2(3.3 \%)$ & \\
\hline ER status & & & $<0.001$ \\
\hline Negative & $152(35.3 \%)$ & $39(63.9 \%)$ & \\
\hline Positive & $278(64.7 \%)$ & $22(36.1 \%)$ & \\
\hline PR status & & & 0.233 \\
\hline Negative & $170(39.5 \%)$ & $29(47.5 \%)$ & \\
\hline Positive & $260(60.5 \%)$ & $32(52.5 \%)$ & \\
\hline HER2 status & & & 0.374 \\
\hline Negative & $293(68.1 \%)$ & $45(73.8 \%)$ & \\
\hline Positive & $137(31.9 \%)$ & $16(26.2 \%)$ & \\
\hline Molecular subtype & & & $<0.001$ \\
\hline Luminal A & $183(42.6 \%)$ & $17(27.9 \%)$ & \\
\hline Luminal B & $95(22.1 \%)$ & $5(8.2 \%)$ & \\
\hline HER2 & $71(16.5 \%)$ & $13(21.3 \%)$ & \\
\hline TNBC & $81(18.8 \%)$ & $26(42.6 \%)$ & \\
\hline
\end{tabular}

Abbreviations: SD, standard deviation; SSBP2, single-stranded DNA-binding protein 2; AJCC, American Joint Committee on Cancer; LN, lymph node; ER, estrogen receptor; PR, progesterone receptor; HER2, human epidermal growth factor receptor 2; TNBC, triple-negative breast carcinoma. ${ }^{\dagger}$ Student's $t$ test. ${ }^{*}$ Cochran-Armitage trend test.

\subsection{Correlations between Nuclear SSBP2 Expression and Patient Outcomes}

We examined the impact of nuclear $S S B P 2$ expression on patient survival. Nine patients with AJCC stage IV were excluded from survival analysis. Patients with negative nuclear SSBP2 expression showed poor prognosis in OS ( $p=0.013$, log-rank test). The patients with negative nuclear SSBP2 expression showed a tendency to have poor RFS, but there was no statistically significant difference $(p=0.175$, log-rank test). The Kaplan-Meier curves for OS and RFS are shown in Figure 2. The univariate Cox regression analysis for OS showed that histological grade $(p=0.002)$, pT stage $(p=0.01)$, ER status $(p=0.009)$, 
lymph node metastasis ( $p=0.002)$, and SSBP2 expression $(p=0.016)$ were significantly associated with OS. The multivariate Cox regression analysis showed that lymph node metastasis ( $p=0.011$ ) was the only independent prognostic factor for OS (Table 3). The subgroup analysis performed according to the molecular subtypes revealed no significant differences in OS or RFS. The Kaplan-Meier curves according to the molecular subtypes are shown in Figures 3 and 4.
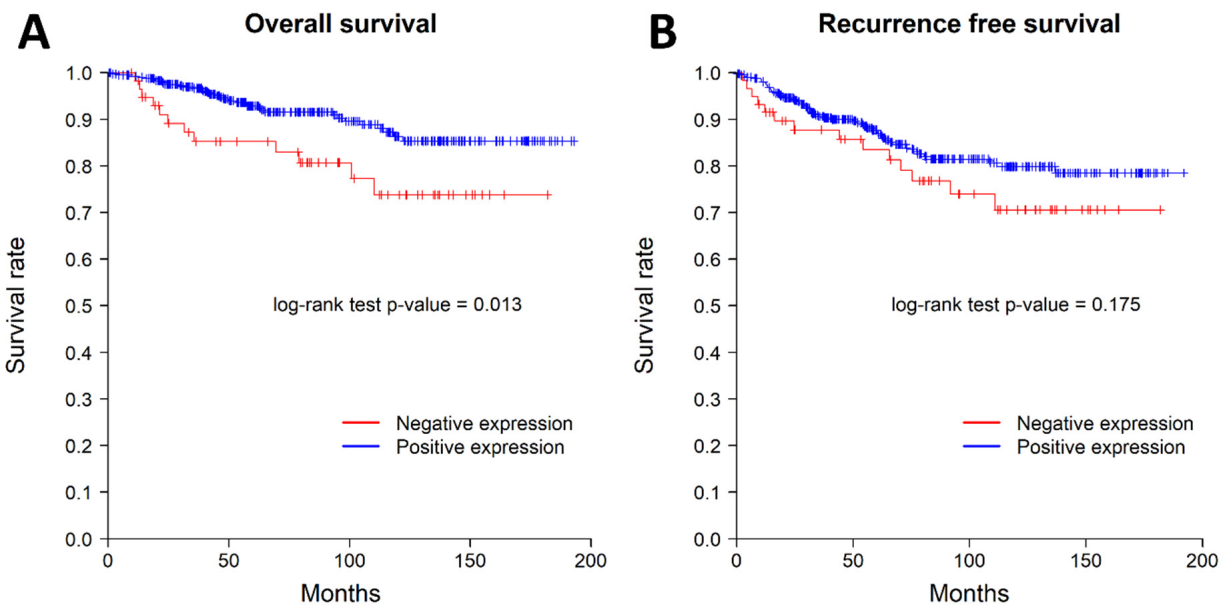

Figure 2. Kaplan-Meier curves for overall survival (A) and recurrence-free survival (B). The patients with loss of nuclear SSBP2 expression showed worse overall survival; however, recurrence-free survival was not associated with nuclear SSBP2 expression.

Table 3. Univariate and multivariate Cox regression analyses for overall survival in patients with breast carcinoma $(n=482)$.

\begin{tabular}{|c|c|c|c|c|c|c|}
\hline & \multicolumn{3}{|c|}{ Univariate Analysis } & \multicolumn{3}{|c|}{ Multivariate Analysis } \\
\hline & HR & $95 \%$ CI & $p$ Value & HR & $95 \%$ CI & $p$ Value \\
\hline Age (per 1 vear) & 0.994 & $0.967-1.021$ & 0.648 & 0.995 & $0.968-1.023$ & 0.723 \\
\hline Histological grade 3 (vs. 1,2) & 2.48 & $1.398-4.4$ & 0.002 & 1.578 & $0.832-2.994$ & 0.163 \\
\hline pT stage 3,4 (vs. 1,2$)$ & 2.701 & $1.262-5.783$ & 0.01 & 1.853 & $0.819-4.192$ & 0.139 \\
\hline ER status positive (vs. negative) & 0.466 & $0.262-0.829$ & 0.009 & 0.627 & $0.324-1.213$ & 0.166 \\
\hline HER2 status positive (vs. negative) & 1.519 & $0.847-2.723$ & 0.161 & 1.35 & $0.742-2.457$ & 0.326 \\
\hline LN metastasis positive (vs. negative) & 2.483 & $1.378-4.474$ & 0.002 & 2.228 & $1.205-4.12$ & 0.011 \\
\hline SSBP2 status negative (vs. positive) & 2.242 & $1.162-4.325$ & 0.016 & 1.942 & $0.97-3.89$ & 0.061 \\
\hline
\end{tabular}

Abbreviations: HR, hazard ratio; CI, confidence interval; vs., versus; SSBP2, single-stranded DNA-binding protein 2; LN, lymph node; ER, estrogen receptor; HER2, human epidermal growth factor receptor 2.
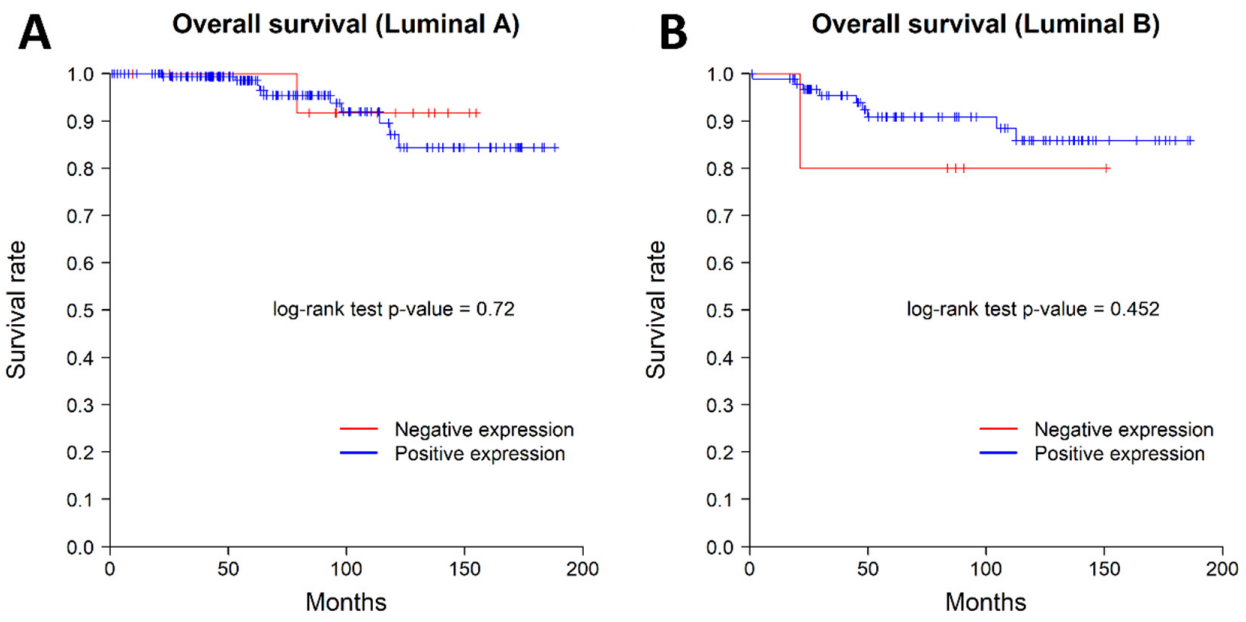

Figure 3. Cont. 

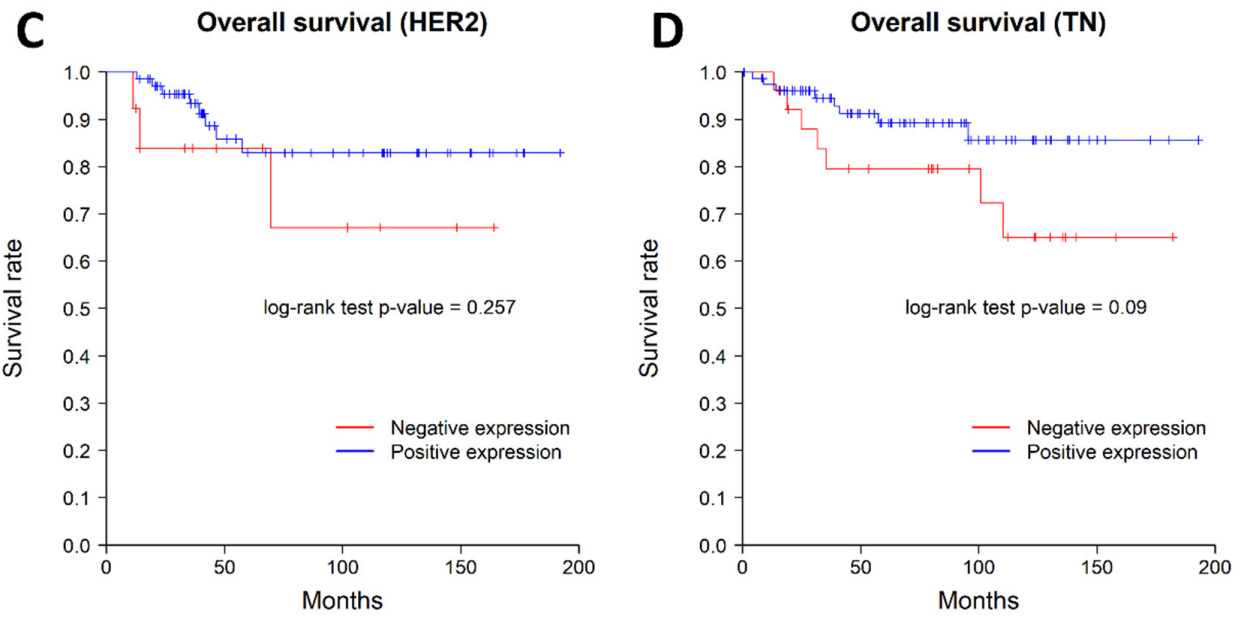

Figure 3. Kaplan-Meier curves for overall survival according to the molecular subtypes. (A) Luminal A type, (B) Luminal B type, (C) HER2 positive type, and (D) triple negative type. There was no difference between the SSBP2-positive and -negative groups in each molecular subtype of breast carcinoma.
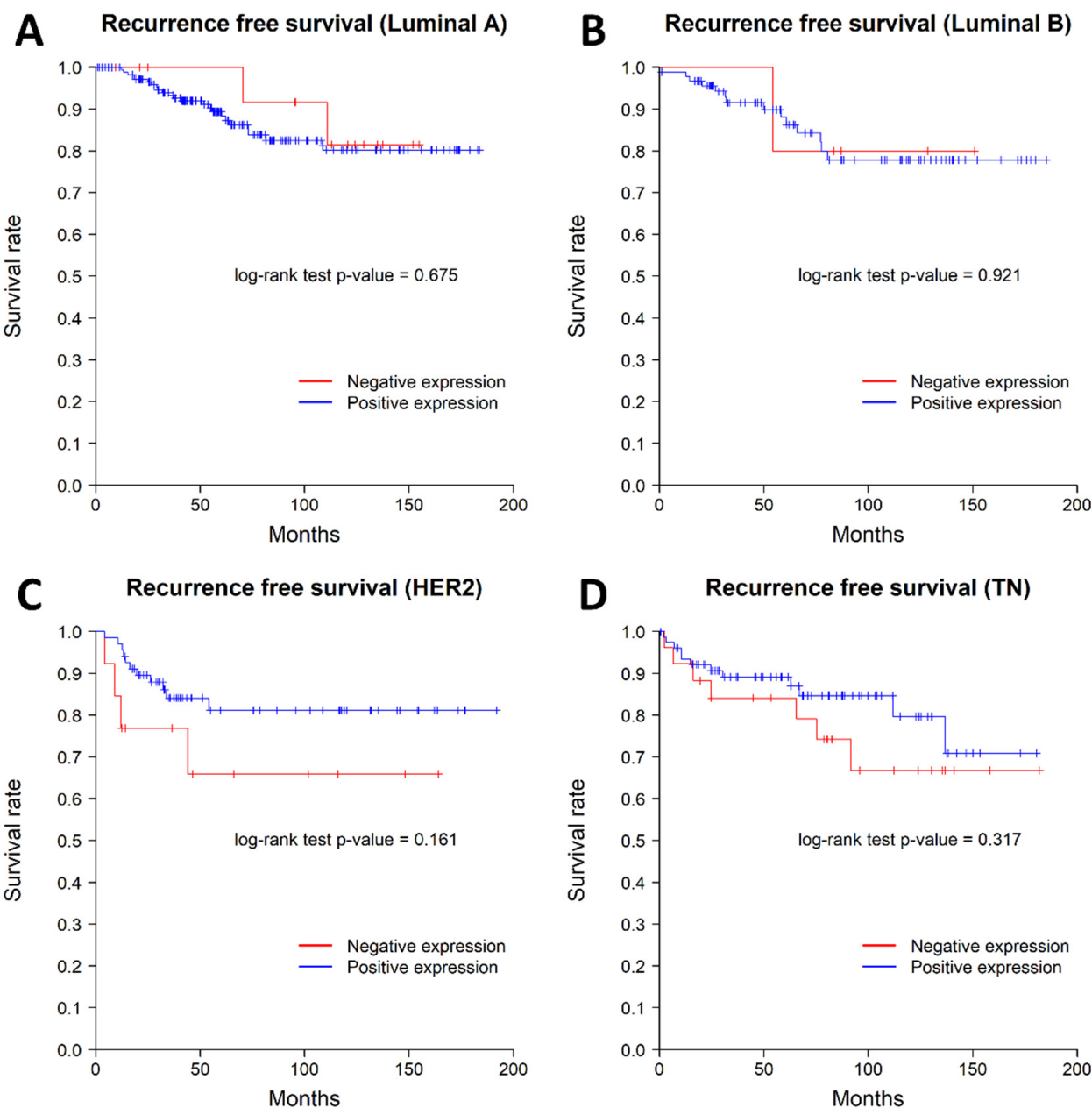

Figure 4. Kaplan-Meier curves for recurrence-free survival according to the molecular subtype. (A) Luminal A type, (B) Luminal B type, (C) HER2 positive type, and (D) triple negative type. There was no difference between the SSBP2-positive and -negative groups in each molecular subtype of breast carcinoma. 


\section{Discussion}

In the present study, we evaluated nuclear SSBP2 expression in 491 cases of invasive breast carcinoma and investigated the correlations between nuclear SSBP2 expression and clinicopathological characteristics and patient survival. Loss of nuclear SSBP2 expression was found in 61 cases of invasive breast carcinoma. Loss of nuclear SSBP2 expression was significantly correlated with larger tumor size, higher histological grade, higher $\mathrm{pT}$ stage, ER status, and molecular subtype. In addition, loss of nuclear SSBP2 expression was associated with poorer OS in patients with invasive breast carcinoma. In the survival analysis according to the molecular subtypes, there was no significant correlation between nuclear SSBP2 expression and OS or RFS.

The human SSBP2 gene was first found in leukemic blasts and is known to be deleted and translocated in acute myelogenous leukemia and myelodysplasia $[15,18,19]$. The SSBP2 gene is one of three related genes with a high level of identity in deduced open-reading frames [10]. The SSBP2 gene is a subunit of a single-stranded DNA binding complex involved in the maintenance of hematopoietic stem cells and stress response, as well as the maintenance of genome stability $[7,20]$. The role of SSBP2 in cancer development and cancer progression appears to vary with the type of malignant tumor. Whether SSBP2 is a tumor suppressor or tumor promoter is still unclear, and there is no consensus on the exact role of SSBP2 expression in human malignancies [2]. There is also no study that supports the exact role of SSBP2 expression in breast cancer until now. Haryono et al. reported that chromosome 5q14.1 with the SSBP2 gene was associated with the risk of breast cancer in a pilot genome-wide association study of breast cancer susceptibility loci in Indonesia [20].

Recently, some studies have suggested that SSBP2 shows a tumor suppressor function in human cancer. Bang et al. reported that loss of nuclear SSBP2 expression was correlated with higher $\mathrm{pT}$ stage, nodal metastasis, and higher AJCC stage in gastric adenocarcinoma. They also found that loss of nuclear SSBP2 expression was associated with poorer RFS in a microsatellite stable and Epstein-Barr virus (EBV)-negative group and HER2-negative group [14]. Chung et al. reported that loss of nuclear SSBP2 expression was observed in $34.3 \%$ of colorectal adenocarcinoma (CRA) and $76.3 \%$ of metastatic CRA cases, and they noted that loss of nuclear SSBP2 expression was associated with higher $\mathrm{pT}$ stage, vascular invasion, and poorer OS in CRA [15]. Kim et al. described loss of nuclear SSBP2 expression in correlation with poor prognostic factors, such as larger tumor size, higher World Health Organization/International Society of Urological Pathology histological grade, tumor necrosis, sarcomatoid change, and higher $\mathrm{pT}$ stage, and noted it was associated with worse RFS in clear cell renal cell carcinoma [16].

Many studies have suggested that $S S B P 2$ is silenced through a molecular pathway mediated by promoter hypermethylation. Liu et al. reported that the SSBP2 promoter was hypermethylated in $61.4 \%$ of prostate cancers, while benign prostatic hyperplasia had no hypermethylation in the SSBP2 promoter [11]. Huang et al. found that SSBP2 promoter methylation and the downregulation of SSBP2 expression are present in $86 \%$ of esophageal squamous cell carcinoma tissue [21]. Brait et al. investigated promoter methylation frequency for 13 genes, including SSBP2, in ovarian cancer, and reported that $9 \%$ of ovarian cancers showed promoter hypermethylation [22]. Finally, Kagohara et al. reported that methylation in the SSBP2 promoter was more frequently identified in adenocarcinoma than cholecystitis of the gallbladder [23].

Some authors have reported that SSBP2 may play a role in promoting cancer progression because its expression was found to be upregulated in some tumors, including glioblastoma and hepatocellular carcinoma [12,13]. Xiao et al. reported that increased SSBP2 expression is statistically associated with poorer OS in patients with glioblastoma [12]. Additionally, Kim et al. found that nuclear SSBP2 expression was associated with tumor multifocality, higher histological grade, vascular invasion, and higher Ki-67 proliferation index, and they also reported that nuclear SSBP2 expression was significantly correlated with poorer OS and RFS in patients with hepatocellular carcinoma [13]. 
In our study, we found that loss of nuclear SSBP2 expression was correlated with larger tumor size, higher histological grade, and higher $\mathrm{pT}$ stage, which indicates that it may be involved in breast cancer progression. During survival analysis, loss of nuclear SSBP2 expression was associated with poorer OS in patients with breast carcinoma. Our results imply that nuclear $S S B P 2$ expression may play a role as a tumor suppressor in breast carcinoma. However, as discussed above, previous studies reported discrepant observations regarding the association of $S S B P 2$ with prognosis. These discrepant reports on $S S B P 2$ raise the demand for further research in the context of various additional biomarkers, which are related to SSBP2.

There are some limitations to our study. First, we used a retrospective study design and included cases that were collected from a single center. In addition, because each case was evaluated as a single core of tissue microarray, it offered a relatively weak representation of the entire lesion. Since the proportion of cases with negative SSBP2 expression is low in our study, routine analysis of $S S B P 2$ expression in every case may have weak clinical utility. However, despite this, it was confirmed that SSBP2 had a significant effect on prognosis in this study. Therefore, further research is needed to identify some subgroups in which SSBP2 has a more significant impact on prognosis or treatment decisions, in combination with additional biomarkers in the future.

In conclusion, we investigated the clinicopathological significance of nuclear SSBP2 expression in 491 invasive breast carcinomas. Loss of nuclear SSBP2 expression was significantly associated with aggressive phenotypes and poorer OS. The exact function of nuclear SSBP2 expression and its potential as a novel biomarker for breast carcinoma should be further evaluated in future studies.

Author Contributions: H.P. performed the experiment, analyzed the data, and wrote the manuscript. S.J., H.S. and H.C. contributed to the collection of pathological data. S.B., H.K. and J.M. revised the manuscript critically for important intellectual content. S.-J.S. constructed the tissue microarray of breast cancer. C.C. and M.S.C. contributed to the collection of clinical data. S.S.P. designed the study and edited and approved the manuscript. All authors have read and agreed to the published version of the manuscript.

Funding: This research was supported by the Basic Science Research Program through the National Research Foundation of Korea (NRF) funded by the Ministry of Education (NRF-2021R1G1A1006465).

Institutional Review Board Statement: This study was approved by the Institutional Review Board of Hanyang University Hospital (HYUH 2021-12-014-001), and the requirement for informed consent was waived.

Informed Consent Statement: Not applicable.

Conflicts of Interest: The authors declare no conflict of interest exist.

\section{References}

1. Sung, H.; Ferlay, J.; Siegel, R.L.; Laversanne, M.; Soerjomataram, I.; Jemal, A.; Bray, F. Global Cancer Statistics 2020: GLOBOCAN Estimates of Incidence and Mortality Worldwide for 36 Cancers in 185 Countries. CA A Cancer J. Clin. 2021, 71, 209-249. [CrossRef] [PubMed]

2. Kim, H.; Jang, S.M.; Ahn, H.; Sim, J.; Yi, K.; Chung, Y.; Han, H.; Rehman, A.; Chung, M.S.; Jang, K.; et al. Clinicopathological Significance of Dual-Specificity Protein Phosphatase 4 Expression in Invasive Ductal Carcinoma of the Breast. J. Breast Cancer 2015, 18, 1. [CrossRef] [PubMed]

3. Jezierska, A.; Motyl, T. Matrix Metalloproteinase-2 Involvement in Breast. Med. Sci. Monit. 2009, 15, RA32-40. [PubMed]

4. Bertolo, C.; Guerrero, D.; Vicente, F.; Cordoba, A.; Esteller, M.; Ropero, S.; Guillen-Grima, F.; Martinez-Peñuela, J.M.; Lera, J.M. Differences and Molecular Immunohistochemical Parameters in the Subtypes of Infiltrating Ductal Breast Cancer. Am. J. Clin. Pathol. 2008, 130, 414-424. [CrossRef]

5. Goldhirsch, A.; Winer, E.P.; Coates, A.S.; Gelber, R.D.; Piccart-Gebhart, M.; Th?rlimann, B.; Senn, H.-J.; Albain, K.S.; Andr?, F.; Bergh, J.; et al. Personalizing the Treatment of Women with Early Breast Cancer: Highlights of the St Gallen International Expert Consensus on the Primary Therapy of Early Breast Cancer 2013. Ann. Oncol. 2013, 24, 2206-2223. [CrossRef]

6. Wang, Y.; Klumpp, S.; Amin, H.M.; Liang, H.; Li, J.; Estrov, Z.; Zweidler-McKay, P.; Brandt, S.J.; Agulnick, A.; Nagarajan, L. SSBP2 Is an in Vivo Tumor Suppressor and Regulator of LDB1 Stability. Oncogene 2010, 29, 3044-3053. [CrossRef] 
7. $\quad$ Li, J.; Kurasawa, Y.; Wang, Y.; Clise-Dwyer, K.; Klumpp, S.A.; Liang, H.; Tailor, R.C.; Raymond, A.C.; Estrov, Z.; Brandt, S.J.; et al. Requirement for Ssbp2 in Hematopoietic Stem Cell Maintenance and Stress Response. J. Immunol. 2014, 193, 4654-4662. [CrossRef]

8. Nishioka, N.; Nagano, S.; Nakayama, R.; Kiyonari, H.; Ijiri, T.; Taniguchi, K.; Shawlot, W.; Hayashizaki, Y.; Westphal, H.; Behringer, R.R.; et al. Ssdp1 Regulates Head Morphogenesis of Mouse Embryos by Activating the Lim1-Ldb1 Complex. Development 2005, 132, 2535-2546. [CrossRef]

9. Zhu, M.; Jiang, B.; Zuo, H.; Wang, X.; Ge, H.; Huang, Z. LIM-Domain-Binding Protein 1 Mediates Cell Proliferation and Drug Resistance in Colorectal Cancer. Front. Surg. 2022, 8, 790380. [CrossRef]

10. Liang, H.; Samanta, S.; Nagarajan, L. SSBP2, a Candidate Tumor Suppressor Gene, Induces Growth Arrest and Differentiation of Myeloid Leukemia Cells. Oncogene 2005, 24, 2625-2634. [CrossRef]

11. Liu, J.-W.; Nagpal, J.K.; Sun, W.; Lee, J.; Kim, M.S.; Ostrow, K.L.; Zhou, S.; Jeronimo, C.; Henrique, R.; Van Criekinge, W.; et al SsDNA-Binding Protein 2 Is Frequently Hypermethylated and Suppresses Cell Growth in Human Prostate Cancer. Clin. Cancer Res. 2008, 14, 3754-3760. [CrossRef] [PubMed]

12. Xiao, Y.; Decker, P.A.; Rice, T.; McCoy, L.S.; Smirnov, I.; Patoka, J.S.; Hansen, H.M.; Wiemels, J.L.; Tihan, T.; Prados, M.D.; et al. SSBP2 Variants Are Associated with Survival in Glioblastoma Patients. Clin. Cancer Res. 2012, 18, 3154-3162. [CrossRef]

13. Kim, H.; Kim, Y.; Chung, Y.; Abdul, R.; Sim, J.; Ahn, H.; Shin, S.-J.; Paik, S.S.; Kim, H.J.; Jang, K.; et al. Single-Stranded DNA Binding Protein 2 Expression Is Associated with Patient Survival in Hepatocellular Carcinoma. BMC Cancer 2018, 18, 1244. [CrossRef] [PubMed]

14. Bang, S.; Kim, H.; Jang, K.; Paik, S.S.; Shin, S.-J. The Loss of Nuclear Expression of Single-Stranded DNA Binding Protein 2 of Gastric Adenocarcinoma and Its Prognostic Role: Analysis of Molecular Subtype. PLoS ONE 2020, 15, e0236896. [CrossRef] [PubMed]

15. Chung, Y.; Kim, H.; Bang, S.; Jang, K.; Paik, S.S.; Shin, S.-J. Nuclear Expression Loss of SSBP2 Is Associated with Poor Prognostic Factors in Colorectal Adenocarcinoma. Diagnostics 2020, 10, 1097. [CrossRef]

16. Kim, H.; Kim, Y.; Bang, S.; Park, S.; Jee, S.; Sim, J.; Shin, S.-J.; Paik, S.S.; Jang, K. Low Expression of Single-Stranded DNA Binding Protein 2 (SSBP2) Predicts Unfavourable Postoperative Outcomes in Patients with Clear Cell Renal Cell Carcinoma. In Vivo 2020, 34, 101-107. [CrossRef]

17. Feeley, L.P.; Mulligan, A.M.; Pinnaduwage, D.; Bull, S.B.; Andrulis, I.L. Distinguishing Luminal Breast Cancer Subtypes by Ki67, Progesterone Receptor or TP53 Status Provides Prognostic Information. Mod. Pathol. 2014, 27, 554-561. [CrossRef]

18. Fairman, J.; Wang, R.; Liang, H.; Zhao, L.; Saltman, D.; Liang, J.; Nagarajan, L. Translocations and Deletions of 5q13.1 in Myelodysplasia and Acute Myelogenous Leukemia: Evidence for a Novel Critical Locus. Blood 1996, 88, 2259-2266. [CrossRef]

19. Castro, P.; Liang, H.; Liang, J.C.; Nagarajan, L. A Novel, Evolutionarily Conserved Gene Family with Putative Sequence-Specific Single-Stranded DNA-Binding Activity. Genomics 2002, 80, 78-85. [CrossRef]

20. Haryono, S.J.; Datasena, I.G.B.; Santosa, W.B.; Mulyarahardja, R.; Sari, K. A Pilot Genome-Wide Association Study of Breast Cancer Susceptibility Loci in Indonesia. Asian Pacific J. Cancer Prev. 2015, 16, 2231-2235. [CrossRef]

21. Huang, Y.; Chang, X.; Lee, J.; Cho, Y.G.; Zhong, X.; Park, I.-S.; Liu, J.-W.; Califano, J.A.; Ratovitski, E.A.; Sidransky, D.; et al Cigarette Smoke Induces Promoter Methylation of Single-Stranded DNA-Binding Protein 2 in Human Esophageal Squamous Cell Carcinoma. Int. J. Cancer 2011, 128, 2261-2273. [CrossRef] [PubMed]

22. Brait, M.; Maldonado, L.; Noordhuis, M.; Begum, S.; Loyo, M.; Poeta, M.L.; Barbosa, A.; Fazio, V.M.; Angioli, R.; Rabitti, C.; et al. Association of Promoter Methylation of VGF and PGP9.5 with Ovarian Cancer Progression. PLoS ONE 2013, 8, e70878. [CrossRef]

23. Kagohara, L.T.; Schussel, J.L.; Subbannayya, T.; Sahasrabuddhe, N.; Lebron, C.; Brait, M.; Maldonado, L.; Valle, B.L.; Pirini, F.; Jahuira, M.; et al. Global and Gene-Specific DNA Methylation Pattern Discriminates Cholecystitis from Gallbladder Cancer Patients in Chile. Future Oncol. 2015, 11, 233-249. [CrossRef] [PubMed] 